\title{
Entrainment of Cellular Circadian Rhythms in Lactuca sativa L. Leaf by Spatially Controlled Illuminations
}

\author{
Naoki Seki ${ }^{1}$, Kazuya Ukai ${ }^{1}$, Takanobu Higashi ${ }^{2}$ and Hirokazu Fukuda ${ }^{1 *}$
}

${ }^{1}$ Department of Mechanical Engineering, Graduate School of Engineering, Osaka Prefecture University, Sakai 599-8531, Japan

${ }^{2}$ Department of Applied Life Sciences, Graduate School of Life and Environmental Sciences, Osaka Prefecture University, Sakai 599-8531, Japan

\begin{abstract}
Plant circadian system works autonomously and responds to various environmental information in cellular level. Conventional studies on controlling of the plant circadian system, however, have not thoroughly considered in cellular level yet. In this study, we investigated spatiotemporal dynamics of cellular circadian rhythms of clock gene CCA1 in leaves that were controlled by the projector lightings in a transgenic lettuce strain AtCCA1::LUC using a bioluminescence imaging. We have succeeded to control the cellular circadian rhythms in the both case of LCD and laser projectors with 24 or $26 \mathrm{~h}$ periods of light-dark cycles. Although light intensity of the laser projector was very small to a required light intensity for growth of lettuces, the circadian rhythm was entrained with high sensitivity for illumination. Our results motivate experimental and theoretical studies of circadian control and development for the highly functional lighting technology in plant productions.
\end{abstract}

Keywords: Circadian rhythm; Clock gene; Oscillators; Phase analysis; Synchronization

\section{Introduction}

Circadian clocks that generate approximately 24-h rhythmicity are present in almost all living organisms. In higher plants, circadian clocks play a crucial role in the regulation of a variety of biological processes, including gene expressions, photosynthesis, and flowering [1]. An important characteristic of the circadian clocks is their entrainment to environmental time cues (zeitgebers), such as changes in external light or temperature [2].

Recent studies have revealed that plant cells act as self-sustained oscillators and interact each other [3-5]. The phase sensitivity of circadian rhythm, therefore, possesses essentially in each plant cell to entrain for environmental cycles. In previous studies, however, the individual-level responses in intact plants were ordinarily investigated $[6,7]$. In addition, the strong pacemaker of circadian system is absent in plant [8]. Therefore, the plant circadian system works as an autonomous distributed system and can show several spatiotemporal dynamics such as spiral wave in Arabidopsis thaliana leaf $[4,5,8,9]$. In plant circadian system, however, there are few studies of spatiotemporal dynamics in cell population levels, despite many studies in molecular and cellular level $[1,10,11]$.

In this study, we tried to control the cellular circadian rhythm spatially in leaves by spatially and temporally controlled illuminations, which are generated by projectors. Spatiotemporal dynamics of cellular circadian rhythms in leaves in a transgenic lettuce strain AtCCA1::LUC were investigated using a bioluminescence imaging. The period of the LCD projector was $24 \mathrm{~h}$ and that of the laser projector was $24 \mathrm{~h}$ or 26 $\mathrm{h}$. The bioluminescent images were taken every $30 \mathrm{~min}$ using higher sensitive cooled CCD camera in the temperature-controlled dark box. The spatiotemporal dynamics of cellular circadian rhythms were investigated from these bioluminescent images.

\section{Materials and Methods}

\section{Plant materials and growth conditions}

Our experiments were carried out using transgenic lettuce (Lactuca sativa L. cv. Greenwave) AtCCA1::LUC, in which an
Arabidopsis thaliana CIRCADIAN CLOCK ASSOCIATED 1 (CCA1) promoter-luc cassette, pABH-CCA1::LUC-C [10], was transformed into Arabidopsis plants via Agrobacterium tumefaciens - mediated transformation [12]. Luciferase protein was synthesized by activating promoter gene AtCCA1, and then bioluminescence was emitted by the chemical reaction with supplied luciferin. This AtCCA1::LUC lettuce was eliminated the bioluminescence, which was proportional to the expression rate of AtCCA1. This bioluminescence showed a circadian rhythm $[13,14]$ and the circadian rhythm could be observed in almost all cells of the leaves even under constant dark condition, as reported in Ukai et al. [9].

AtCCA1::LUC plants were grown in hydroponic culture (Otsuka-A; Otsuka Co., Ltd., Japan)) under light/dark cycles using fluorescent light with about $150 \mu \mathrm{mol} \mathrm{m}^{-2} \mathrm{~s}^{-1}$ (photosynthesis photon flux) for $3 \sim 5$ weeks. Young leaves in the plants were detached and set on a dish $(40 \mathrm{~mm}$ in diameter), then about $5 \mathrm{ml}$ of $0.2 \mathrm{mM}$ luciferin solution dissolved in water was poured in the same dish.

\section{Illumination conditions and monitoring bioluminescence}

To control the cell-level circadian rhythm in leaf, we applied a spatially controlled illumination for the leaves. Illumination with a set of star-shaped patterns, a bright star within a dark rectangle and its inverted image, was applied using a liquid crystal display (LCD) projector (EB-1915, SEIKO-EPSON KK, Japan) or a scanning laser projector (SHOWWX, MicroVision Inc., USA) at $t_{e}=0$ (Figures 1a and $1 \mathrm{~b}$ ). The spectrum of the super-high pressure mercury vapor lamp, a light source of the LCD projector, shows a sharp peak at 435.8

*Corresponding author: Hirokazu Fukuda, Department of Mechanical Engineering, Graduate School of Engineering, Osaka Prefecture University, Sakai 599-8531, Japan, Tel: +81-72-254-791; E-mail: fukuda@me.osakafu-u.ac.jp

Received September 11, 2015; Accepted October 17, 2015; Published December 01, 2015

Citation: Seki N, Ukai K, Higashi T, Fukuda H (2015) Entrainment of Cellular Circadian Rhythms in Lactuca sativa L. Leaf by Spatially Controlled Illuminations. J Biosens Bioelectron 6: 186. doi:10.4172/2155-6210.1000186

Copyright: ( 2015 Seki N, et al. This is an open-access article distributed under the terms of the Creative Commons Attribution License, which permits unrestricted use, distribution, and reproduction in any medium, provided the original author and source are credited. 
a

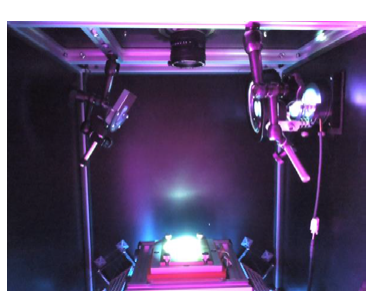

b

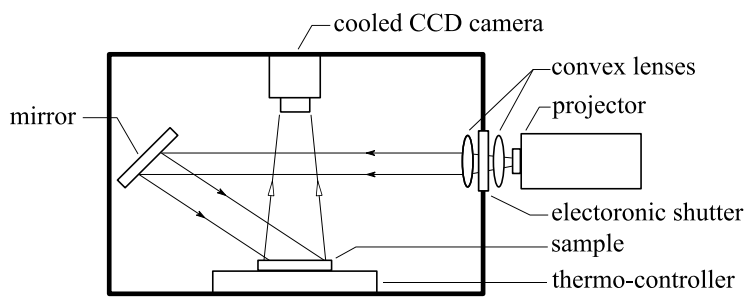

C

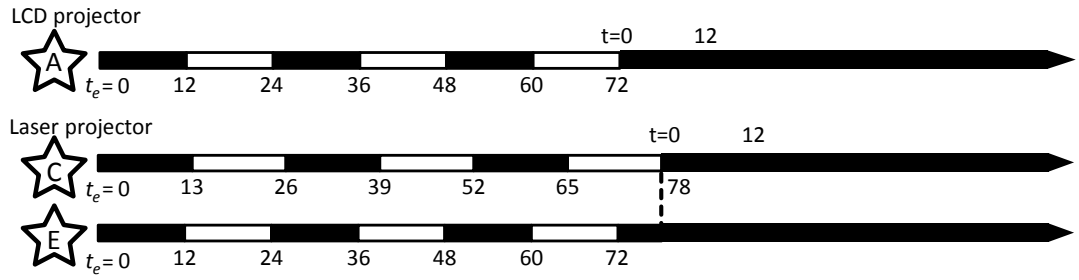

Figure 1: Phase control system of the cellular circadian rhythms. (a) The photograph of the phase control system. (b) The schematic design of the phase control system. Light was reflected in the sputtering mirror through multiple lenses, and it was projected on the sample on the thermo-controller. (c) Illumination protocol of LCD and laser projector. The start time of the entrainment and measurement were at $t=0 \mathrm{~h}$ and $t=0 \mathrm{~h}$, respectively. The star labelled with A, C and E explained the illumination period of the star-shaped pattern in Figures $2 \mathrm{e}$ and $3 \mathrm{~d}$. The white and black bar before $t=0$ show the bright and dark star-shaped pattern, respectively. Subsequently, black bar shows DD condition.

(G-line), 404.7 (H-line) and 365.4 (I-line) $\mathrm{nm} \mathrm{[15]} \mathrm{and} \mathrm{the} \mathrm{spectrum}$ spreads broadly between 350 to $500 \mathrm{~nm}$, while that of the scanning laser projector shows three sharp peaks at 442, 532 and $642 \mathrm{~nm}$. The frequency of projection was $60 \mathrm{~Hz}$ both in the LCD and scanning laser projectors.

In this study, to demonstrate finally the precise entrainment of cellular circadian rhythms by laser projector illuminations we performed two entrainment-protocols: The bright and dark star images were alternately applied with $12 \mathrm{~h}$ or $13 \mathrm{~h}$ periods (Figure 1c). The difference of alternation periods $(12 \mathrm{~h}$ or $13 \mathrm{~h}$ ) in the laser projector experiment will provide the different initialized phases, because of the different start time of continuous dark (DD) condition $(t=-6 \mathrm{~h}$ in the right star, $t=0 \mathrm{~h}$ in the left star).

In the experiment using LCD projector, the alternation period of bright and dark star images was $12 \mathrm{~h}$, and the light intensity of the bright and dark regions were 70 and $1 \mu \mathrm{mol} \mathrm{m} \mathrm{m}^{-2} \mathrm{~s}^{-1}$, respectively (Figure 2a). On the other hand, in the experiment using the laser projector, the alternation period was 12 or $13 \mathrm{~h}$ in the right or left half, and the bright and dark regions were 3 and $0 \mu \mathrm{mol} \mathrm{m} \mathrm{m}^{-2} \mathrm{~s}^{-1}(70$ and 0 $\mu \mathrm{W}$ ), respectively (Figure 3a). In both experiments, the illumination was projected on a detached leaf in the dish (in diameter $40 \mathrm{~mm}$ ) on the thermo-controller (OKS-C201, OKANO CABLE Co., Ltd., Japan) at $22.0 \pm 0.1^{\circ} \mathrm{C}$. Bioluminescence of detached leaves was started to monitor with a highly sensitive cooled CCD camera (ORCA-AG, Hamamatsu Photonics KK, Japan) every $30 \mathrm{~min}$ at $t=0$. The resolution of bioluminescence images was 100 and $200 \mu \mathrm{m}$ in the experiments using LCD and laser projector, respectively.

\section{Results}

Entrainment of cellular circadian rhythm by an LCD projector illumination

Leaves of transgenic lettuce AtCCA1::LUC showed circadian oscillations of bioluminescence under DD as reported by Ukai et al. [9]. Figure $2 \mathrm{~b}$ (right and left panels) shows bioluminescence images under $\mathrm{DD}$ at $t=2.5 \mathrm{~h}$ and $14.5 \mathrm{~h}$. White and black star patterns were observed in the bioluminescence, indicating that their phase was almost the inverse of each other. The star pattern region remained for at least two days, though the intensity of bioluminescence rapidly decreased in time (Figure 2c). To investigate precisely the cellular entrainment of the circadian rhythm in the leaf, we introduced the phase of the circadian oscillation which is determined by following equation $[4,8,9]$.

$$
\phi(t)=2 \pi \frac{t-\tau_{k}}{\tau_{k+1}-\tau_{k}}, t \in\left[\tau_{k}, \tau_{k+1}\right)
$$

Where $\tau_{k}$ is the time of the $k$ th peak of the oscillatory time series of bioluminescence in each pixel. To calculate peaks of bioluminescence oscillation, which often showed large noise, the moving average with a window size of $24 \mathrm{~h}$ window was applied in each pixel. Figure $2 \mathrm{~d}$ shows the phase images of the corresponding circadian bioluminescence in Figure 2c. Figure $2 \mathrm{e}$ shows that enlarged phase images at $t=31$ and 43 $h$. The star pattern emerged very clearly in the bioluminescence and phase images, which means that the phase was very finely initialized with the star form by the LCD illumination (Movie S1). Figure $2 \mathrm{f}$ shows the average of circadian rhythms in the star region (A) and its neighbor (B) in Figure 2e, which were extracted the long-term trend (exponential decay in this case) of bioluminescence intensity. The first peak of the region (A) and (B) was $15 \mathrm{~h}$ and $24 \mathrm{~h}$ from turning off the light, respectively. The peak of the AtCCA1::LUC signal was delay for about $2 \mathrm{~h}$ after turning on the illumination, that is, $14 \mathrm{~h}$ after turning off the illumination $[16,17]$. Under above consideration, these peaks of the region (A) and (B) showed, respectively, $1 \mathrm{~h}$ delay and $2 \mathrm{~h}$ advance compared with the expectation. The phase in the region (A) was almost reversed to the region (B) with a delay of about $9 \mathrm{~h}$. From the results, we succeed to entrain the cellular circadian rhythm by a spatially controlled illumination using an LCD projector. 
a

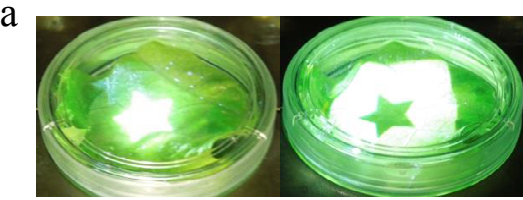
$\mathrm{t}_{\mathrm{e}}=\mathrm{Oh}$

$\mathrm{C}$

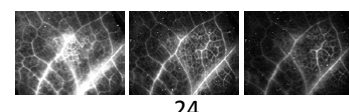

d

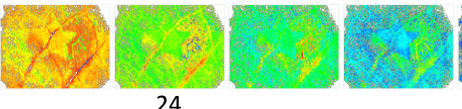

24

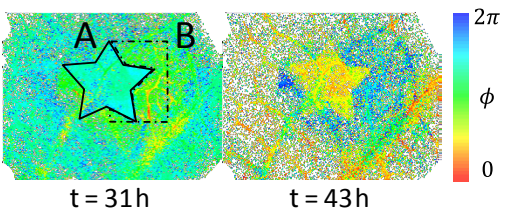

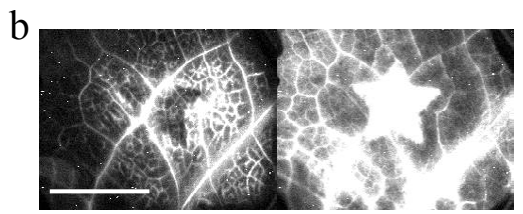

$\mathrm{t}=2.5 \mathrm{~h}$

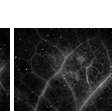

2. $\times 8$

36

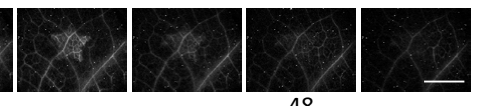

48

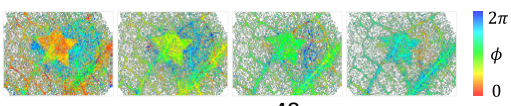

48

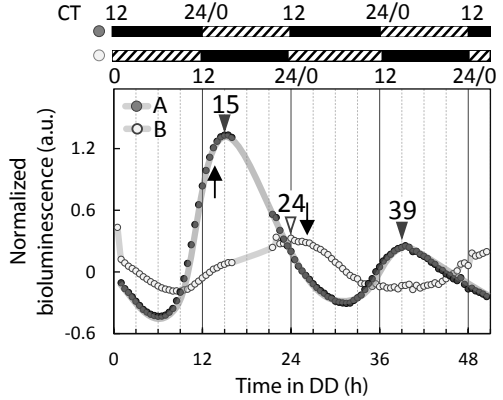

Figure 2: Circadian rhythms with star-shaped initial conditions in an AtCCA1:: LUC lettuce leaf induced by spatiotemporal illumination using an LCD projector. (a) Bright and dark star pattern illumination. (b) Bioluminescence images: the star region was at subject dusk (left) and subject dawn (right). (Scale bar: $10 \mathrm{~mm}$ ). (c) Snapshots of bioluminescence under DD. (Interval between images $=4 \mathrm{~h}$, scale bar $=10 \mathrm{~mm}$ ) $(\mathrm{d})$ Phase images obtained from bioluminescence images of the leaf in Figure 2c. (Interval between images $=4 \mathrm{~h}$ ) (e) Inversion phase images of the leaf of Figure $2 \mathrm{c}$. $t=31$ (left) and $43 \mathrm{~h}$ (right), respectively. (f) Normalized bioluminescence in the star region (black) and the region to its right (white), indicated by the solid line $(A)$ and dashed-dotted line $(B)$ in Figure 2e. There were disturbances in $t=16$ to 21 . Black and white triangles indicated measured peak time of the normalized bioluminescence and arrows indicated expected peak time.Black bars: subjective night; hatched bars: subjective day. The lines were used only to guide the eye.

\section{Entrainment of cellular circadian rhythm by a laser projector illumination}

To demonstrate the precise entrainment of cellular circadian rhythms by laser projector illuminations we performed two entrainment-protocols: The bright and dark star images were alternately applied with $12 \mathrm{~h}$ or $13 \mathrm{~h}$ periods (Figure 1c). The simultaneously application of dual periods ( $12 \mathrm{~h}$ and $13 \mathrm{~h}$ ) for one leaf will provide the different initialized phases by the different start-time of DD condition ( $t=-6 \mathrm{~h}$ in the right star, $t=0 \mathrm{~h}$ in the left star in Figure 1c). The center dash-dotted line in Figure 3a shows the boundary line between $12 \mathrm{~h}$ and $13 \mathrm{~h}$ period regions. Figures $3 \mathrm{~b}$ and $3 \mathrm{c}$ show the bioluminescence and its corresponding phase images. The star patterns were not clearly emerged in the bioluminescence images (Figure $3 \mathrm{~b}$ ) but were emerged in phase images (Figure 3c). The elimination of temporal noise of bioluminescence by the moving average provided successfully the pattern extraction on the phase images. Figure $3 \mathrm{~d}$ shows that enlarged phase images at $t=12.5$ and $24.5 \mathrm{~h}$. The shape of the star region remained for at least one day, in spite of the rapid decrease of bioluminescence. Figure $3 \mathrm{e}$ shows the time series of normalized bioluminescence of these four regions (C, D, E and F). Because the peak of the AtCCA1::LUC bioluminescence oscillation was observed after $2 \mathrm{~h}$ from turning on the illumination, we can estimate the peak-time of the oscillation even under DD, as shown as the arrows in Figures $3 \mathrm{e}$ and $3 \mathrm{f}$.

In the experiment for $24 \mathrm{~h}$ period illumination, the peak of the oscillation in the regions (E) and (F) was at $16 \mathrm{~h}$ and 27 after turning off the illumination, respectively. Although these times were delayed with $2 \mathrm{~h}$ and $1 \mathrm{~h}$ for the expected peak-time (the arrows in Figure $3 \mathrm{f})$, they were almost consistent with the expectation. The regions (E) and $(\mathrm{F})$ were reversed each other with $11 \mathrm{~h}$ time difference. On the other hand, in the experiment for $26 \mathrm{~h}$ period illumination, the peak of the oscillation in the regions (C) and (D) was at 12 and $21 \mathrm{~h}$ after turning of the illumination, respectively. These times were advanced with about $2 \mathrm{~h}$ or $5 \mathrm{~h}$ for the expected peak-times (the arrows in Figure $3 \mathrm{e})$. The regions (C) and (D) were almost reversed each other with 9 $\mathrm{h}$ time difference. The $26 \mathrm{~h}$ period entrainment protocol had worse precision than that of $24 \mathrm{~h}$ period. This failure might be caused by the mismatch between periods of light-dark cycles $(26 \mathrm{~h})$ and circadian clock (approximately $24 \mathrm{~h}$ ).

\section{Discussion}

As shown in bioluminescence and phase images, the circadian oscillation in leaf was not homogeneous [9]. In particular, the vein showed brighter bioluminescence refer to surround one (Figures $2 b$ and $3 \mathrm{~b}$ ), indicating that the constituent surrounding cells of vascular bundles activate the AtCCA1 gene expression. The mature vein cells which have no AtCCA1 genes cannot generate circadian rhythm. Therefore, phase delay in the vein was observed as reported in our previous works $[4,9]$. Moreover, the circadian oscillation in detached 

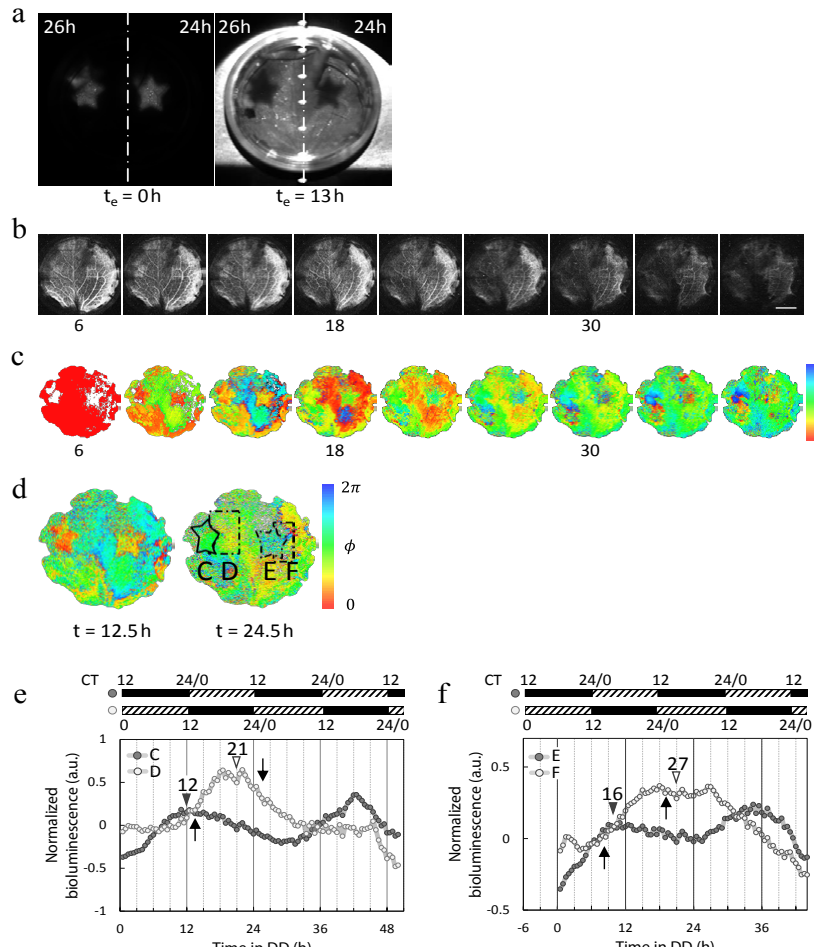

Figure 3: Circadian rhythms with different two star-shaped initial conditions in an AtCCA1::LUC lettuce leaf induced by spatiotemporal illumination using scanning laser projector. (a) Bright and dark star pattern illumination. Dash dotted line showed the boundary two illumination protocols: LD24 (right) and LD26 (left). (b) Snapshots of bioluminescence under DD (Interval between images $=4 \mathrm{~h}$, scale bar $=10 \mathrm{~mm}$ ). (c) Phase image of bioluminescence in the leaf of Figure $3 \mathrm{~b}$ (Interval between images $=4 \mathrm{~h}$ ). (d) Inversion phase images of bioluminescence in the leaf of Figure 3b. $t=12.5$ (left) and $24.5 \mathrm{~h}$ (right), respectively. $(e, f)$ Normalized bioluminescence in the star region $\mathrm{C}$ and $\mathrm{E}$ (gray) and the region to its right ( $\mathrm{D}$ and $\mathrm{F}$ ) (white), indicated by the lines in Figure $3 \mathrm{~d}$ with peak time which was elapsed time since $72 \mathrm{~h}$ after applying illumination.Gray and white triangles indicated measured peak time of the normalized bioluminescence and arrows indicated expected peak time. Black bars: subjective day; hatched bars: subjective night.

leaf showed the rapid decay of cellular bioluminescence, which breaks the entrained pattern. Therefore, it was hard to control cellular circadian rhythm with high homogeneity and sustainability in our experiments. The establishment of methodology for homogeneous and sustainability entrainment should be considered in future work.

Our LCD projector system could provide sufficiently strong illumination (maximally $70 \mu \mathrm{mol} \mathrm{m} \mathrm{m}^{-2} \mathrm{~s}^{-1}$ ) but it has multiple assignments: The power consumption of our LCD projector system was $340 \mathrm{~W}$ for photosynthetic photon flux density (PPFD) $70 \mu \mathrm{mol}$ $\mathrm{m}^{-2} \mathrm{~s}^{-1}$. The illumination efficiency (light intensity per electric power) of the LCD projector was about 23 and 35 times compared with the FL $(15 \mathrm{~W})$ and the LEDs $(10 \mathrm{~W})$, that is, the LCD projector system was very high cost. Therefore, the low power consumption LCD projector is required to decrease the lighting-cost. The high contrast between illuminated and blank regions is also demanded in order to control the circadian clock accurately, but in our LCD system the light intensity of the blank region was $1 \mu \mathrm{mol} \mathrm{m} \mathrm{m}^{-2} \mathrm{~s}^{-1}$. On the other hand, in our scanning laser projector system, although the laser illumination was significantly weak (maximally $3 \mu \mathrm{mol} \mathrm{m} \mathrm{m}^{-2} \mathrm{~s}^{-1}$ ) to live through photosynthesis, it entrained successfully the cellular circadian rhythms and printed the reverse phase pattern on the leaves as shown in Figure 3c. From this result, the circadian clock is very sensitive for light cues so that it is able to control the cellular circadian rhythm by a scanning laser projector. Moreover, the scanning laser projector has high illumination efficiency and high contrast of illumination of which blank region is completely dark.

There are horticultural meanings in the spatially control of circadian rhythms as follows: The circadian clock gene is upstream of the FLOWERING LOCUS T $(F T)$ involved in floral induction $[18,19]$. Floral control is expected by regulation of the FT gene expression using local projection such as changing the light quality or period depending on the individual organs. The influence on the plant growth is different relying on the wavelength [20]. Therefore, spatially-controlled illumination with optimal wavelengths and day-lengths for significant organs might increase plant growth and quality.

\section{Conclusions}

We showed that the circadian rhythm of lettuce leaf could be controlled spatially by using an LCD or a scanning laser projector. The inversed region of cellular circadian rhythm remained for at least two days, in spite of the rapid decrease of bioluminescence. Although light intensity of the laser projector was very small to a required light intensity for growth of lettuces, the cellular circadian rhythm was successfully controlled. Therefore, the projector illumination is useful to regulate the plant metabolism spatially through the controlling cellular circadian rhythm.

\section{Acknowledgements}

This study was partially supported by Grants-in-Aid for Scientific Research (No. 25712029 and 25119721 to H.F.) and the research foundation for OptoScience and Technology.

\section{References}

1. McClung CR (2006) Plant circadian rhythms. Plant Cell 18: 792-803.

2. Johnson $\mathrm{CH}$, Elliott JA, Foster R (2003) Entrainment of circadian programs. Chronobiol Int 20: 741-774

3. Thain SC, Hall A, Millar AJ (2000) Functional independence of circadian clocks that regulate plant gene expression. Current Biology: CB 10: 951-956.

4. Fukuda H, Nakamichi N, Hisatsune M, Murase H, Mizuno T (2007) Synchronization of plant circadian oscillators with a phase delay effect of the vein network. Phys Rev Lett 99: 098102.

5. Fukuda H, Ukai K, Oyama T (2012) Self-arrangement of cellular circadian rhythms through phase-resetting in plant roots. Phys Rev E Stat Nonlin Soft Matter Phys 86: 041917.

6. Ohara T, Fukuda H, Tokuda IT (2015) Phase response of the Arabidopsis thaliana circadian clock to light pulses of different wavelengths. J Biol Rhythms 30: 95-103.

7. Ohara T, Fukuda H, Tokuda IT (2015) An extended mathematical model for reproducing the phase response of Arabidopsis thaliana under various light conditions. Journal of Theoretical Biology 382: 337-344.

8. Wenden B, Toner DL, Hodge SK, Grima R, Millar AJ (2012) Spontaneous spatiotemporal waves of gene expression from biological clocks in the leaf. Proc Natl Acad Sci USA 109: 6757-6762.

9. Ukai K, Inai K, Nakamichi N, Ashida H, Yokota A, et al. (2012) Traveling Waves of Circadian Gene Expression in Lettuce. Environmental Control in Biology 50: 237-246.

10. Nakamichi N, Ito S, Oyama T, Yamashino T, Kondo T, et al. (2004) Characterization of plant circadian rhythms by employing Arabidopsis cultured cells with bioluminescence reporters. Plant and Cell Physiology 45: 57-67.

11. McClung CR, Gutierrez RA (2010) Network news: prime time for systems biology of the plant circadian clock. Curr Opin Genet Dev 20: 588-598.

12. Bechtold N, Ellis J, Pelletier G (1993) In planta Agrobacterium mediated gene 
Citation: Seki N, Ukai K, Higashi T, Fukuda H (2015) Entrainment of Cellular Circadian Rhythms in Lactuca sativa L. Leaf by Spatially Controlled Illuminations. J Biosens Bioelectron 6: 186. doi:10.4172/2155-6210.1000186

Page 5 of 5

transfer by infiltration of adult Arabidopsis thaliana plants. C R Acad Sci Ser III Sci Vie Life Sci. Sciences Paris Life Sciences 316: 1194-1199.

13. Higashi T, Kamitamari A, Okamura N, Ukai K, Okamura K, et al. (2014) Characterization of Circadian Rhythms Through a Bioluminescence Reporter Assay in Lactuca sativa L. Environmental Control in Biology 52: 21-27.

14. Higashi T, Nishikawa S, Okamura N, Fukuda H (2015) Evaluation of Growth under Non-24 h Period Lighting Conditions in Lactuca sativa L. Environment Control in Biology 53: 7-12.

15. Smith BW, Suzuki K (2007) Microlithography: Science and Technology, (2ndedn), Taylor and Francis.

16. Fukuda $H$, Ichino $T$, Kondo $T$, Murase $H$ (2011) Early diagnosis of productivity through a clock gene promoter activity using a luciferase bioluminescence assay in Arabidopsis thaliana. Environmental Control in Biology 49: 51-60.
17. Fukuda $\mathrm{H}$, Uchida $\mathrm{Y}$, Nakamichi $\mathrm{N}$ (2008) Effect of a dark pulse under continuous red light on the Arabidopsis thaliana circadian rhythm. Environmental Control in Biology 46: 123-128.

18. Suárez-López P, Wheatley K, Robson F, Onouchi H, Valverde F, et al. (2001) CONSTANS mediates between the circadian clock and the control of flowering in Arabidopsis. Nature 410: 1116-1120.

19. Endo M, Shimizu H, Nohales MA, Araki T, Kay SA (2014) Tissue-specific clocks in Arabidopsis show asymmetric coupling. Nature 515: 419-422.

20. Lin KH, Huang MY, Huang WD, Hsu MH, Yang ZW, et al. (2013) The effects of red, blue, and white light-emitting diodes on the growth, development, and edible quality of hydroponically grown lettuce (Lactuca sativa $L$ var capitata). Scientia Horticulturae 150: 86-91. 